\title{
Equilibrium States for a Classical Lattice Gas
}

\author{
H. J. BRASCAMP \\ Institute for Theoretical Physics, Rijks-Universiteit, Groningen, the Netherlands
}

Received March 9, 1970

\begin{abstract}
Various definitions of thermodynamic equilibrium states for a classical lattice gas are given and are proved to be equivalent. In all cases, a set of equations is given, the solutions of which are by definition equilibrium states. Examples are the condition of Lanford and Ruelle, and the KMS boundary condition. In connection with this, it is shown that the time translation for classical interactions exists as an automorphism of the quantum algebra of observables, under conditions which are weaker than those found for quantum interactions.
\end{abstract}

\section{Introduction}

In this paper, various definitions of a thermodynamic equilibrium state for a classical lattice gas will be compared.

According to the grand canonical prescription, the equilibrium state for given temperature and chemical potential is found as the limit of finite volume Gibbs states. In some cases however (phase transitions) the limit is not unique. Therefore one may try to define equilibrium states directly for the infinite system. There are two ways known.

1. The Variational Principle. An equilibrium state is a translationally invariant state, that maximizes the pressure [5]. By the occurrence of the mean entropy, translations play an essential role.

2. The KMS-condition and other Equivalent Conditions. A set of equations is given, solutions of which are by definition equilibrium states.

In the present paper we will concentrate on the second way. We will find, that it works independent of the presence, if any, of lattice translations. Therefore they will not be taken into account.

In Section 4, a number of equivalent equilibrium conditions are given. One of them is a condition by Lanford and Ruelle [7] and Dobrushin $[12,13]$. When restricted to invariant states, this condition is equivalent to the variational principle [7].

In Section 6, the KMS-condition is shown to be equivalent to the conditions of Section 4.

As a preliminary step to the KMS-condition, time translations are studied in Section 5. In order to get a non-trivial automorphism, we have to imbed the classical algebra into the quantum algebra (Section 2). 
The automorphism is found to exist for interactions satisfying Eq. (1). This condition is somewhat weaker than those by Robinson [3], Ruelle ([1], p. 192), and Manuceau and Trotin [10]. However, these authors treat the much more general case of quantum interactions.

In Section 7, limits of Gibbs states are shown to satisfy the KMScondition. In Section 8, the connection with the Gallavotti-Miracle [9] and Kirkwood-Salsburg equations is given.

Let us give now some definitions. In the present paper, our lattice will be nothing but a countable set of points. Usually, one takes a $v$ dimensional lattice, in which case there are $v$ independent vectors that map the lattice onto itself. In the present paper, however, lattice translations don't play any role. If they are not considered, the word "dimension" is meaningless.

The lattice points can be empty or occupied with at most one particle. To a set $X$ of $n$ occupied points there corresponds an $n$-body potential $\Phi(X)$; for the classical lattice gas, $\Phi(X)$ is just a real number $[4,5,7,9]$. So now an interaction is defined as a mapping $\Phi$ from the finite sets $X \subset Z$ into the real numbers. The following conditions are imposed on $\Phi$ (cf. $[7,9])$

1. $\Phi(\emptyset)=0$.

2. $\sum_{x \in X}|\Phi(X)| \equiv\|\Phi\|_{x}<\infty$, for all $x \in Z$.

In one case (Section 8) we will need an extra condition

3. $\sup _{x \in Z}\|\Phi\|_{x} \equiv\|\Phi\|<\infty$.

Again, usually $\Phi$ is taken translationally invariant. In that case, $\|\Phi\|_{x}$ is independent of $x$ and Condition 3 is automatically fulfilled.

We will say that an interaction $\Phi$ has finite range, if for any $x \in Z$ there is a finite set $\Lambda(x)$ such, that

$$
\Phi(X)=0 \quad \text { if } \quad x \in X, X \notin \Lambda(x) .
$$

A finite range interaction satisfies Condition 2.

The total energy of a set $X$ of occupied points is

$$
U(X)=\sum_{S \subset X} \Phi(S) .
$$

In the following, equilibrium states for the infinite lattice, belonging to the interaction $\Phi$, will be considered. For a finite sublattice $\Lambda$ the correlation functions $\varrho^{(\Lambda)}(X), X \subset \Lambda$, according to Gibbs are

$$
\varrho^{(\Lambda)}(X)=\left[\sum_{Y \subset A} e^{-U(Y)}\right]^{-1} \sum_{X \subset Y \subset A} e^{-U(Y)} .
$$


The factor $\beta$ (inverse temperature) and the term $-N(Y) \mu$ (chemical potential) are absorbed in the energy $U(Y)$. The various definitions of infinite volume equilibrium states are based on this prescription.

When considering the thermodynamical limit, we will write

if

$$
\lim _{\Lambda \rightarrow \infty} f(\Lambda)=f
$$

$$
\lim _{n \rightarrow \infty} f\left(\Lambda_{n}\right)=f,
$$

for any sequence $\left\{\Lambda_{n}\right\}$ with the properties

1. $\Lambda_{n+1} \supset \Lambda_{n}$.

2. For any finite $M \subset Z$ there is an integer $m$ such that $\Lambda_{m} \supset M$.

\section{Observables}

In this section we will briefly repeat the definitions of the quantum algebra of observables and the classical algebra of observables. We will indicate how the classical algebra can be considered as a subalgebra of the quantum algebra. Let us start with the quantum algebra $[1,2,3]$.

With each lattice point $x \in Z$ there is associated a two dimensional Hilbert space $\mathfrak{H}_{x}$. Observables in $x$ are bounded operators in $\mathfrak{H}_{x}$. There are four independent ones; let us take the creation, annihilation and particle number operators

They satisfy

$$
a(x), a^{*}(x), a^{*}(x) a(x), a(x) a^{*}(x) .
$$

$$
a^{*}(x) a(x)+a(x) a^{*}(x)=1 .
$$

To a finite set $\Lambda \subset Z$ there correspond the Hilbert space

$$
\mathfrak{H}_{\Lambda}=\bigotimes_{x \in \Lambda} \mathfrak{H}_{x}
$$

and the algebra of observables in $\Lambda$

$$
\mathfrak{a}(\Lambda)=\mathfrak{B}\left(\mathfrak{H}_{A}\right) .
$$

A basis for $\mathfrak{H}_{A}$ is

$$
\left\{a^{*}(X) \Omega, X \subset \Lambda\right\},
$$

where $\Omega$ is the vacuum state, and

$$
a^{*}(X)=\bigotimes_{x \in X} a^{*}(x) .
$$


If $\Lambda_{1} \subset \Lambda_{2}$, the algebra $\mathfrak{a}\left(\Lambda_{1}\right)$ can be identified with $\mathfrak{a}\left(\Lambda_{1}\right) \otimes 1_{\Lambda_{2} / \Lambda_{1}}$; in that sense

$$
\mathfrak{a}\left(\Lambda_{1}\right) \subset \mathfrak{a}\left(\Lambda_{2}\right) .
$$

If $\Lambda_{1} \cap \Lambda_{2}=\emptyset$, the algebras $a\left(\Lambda_{1}\right)$ and $a\left(\Lambda_{2}\right)$ commute.

Due to the isotony relation (7), one can define

$$
\mathfrak{a}_{L}=\bigcup_{\Lambda} \mathfrak{a}(\Lambda) ; \mathfrak{a}=\overline{\mathfrak{a}}_{L} .
$$

The $C^{*}$-algebra of observables $\mathfrak{a}$ is the norm closure in $\mathfrak{B}\left(\mathfrak{H}_{Z}\right)$ of the algebra of local observables $\mathfrak{a}_{L}$.

Let us now consider the classical algebra of observables, as defined in [4-6]. An observable in $\Lambda$ is a function $A(X)$ of all subsets $X \subset \Lambda$, or equivalently, a function $A(X)$ satisfying

$$
A(X)=A(X \cap \Lambda), X \subset Z .
$$

It can be identified with the operator

$$
A=\sum_{X \subset \Lambda} A(X) a^{*}(X) a(X) a(\Lambda / X) a^{*}(\Lambda / X),
$$

which satisfies

$$
\left(a^{*}(X) \Omega, A a^{*}(Y) \Omega\right)=A(X) \delta_{X Y} .
$$

So in the representation given by the basis vectors (6), $A$ is a diagonal matrix with diagonal elements $A(X)$. Its norm is then

$$
\|A\|=\sup _{X \subset A}|A(X)| .
$$

Now a classical observable is a function $A(X)$ with

$$
A(X)=\lim _{\Lambda \rightarrow \infty} A(X \cap \Lambda),
$$

uniformly in $X$. It corresponds to the quantum observable

$$
A=\lim _{\Lambda \rightarrow \infty} \sum_{X \subset \Lambda} A(X) a^{*}(X) a(X) a(\Lambda / X) a^{*}(\Lambda / X) .
$$

According to Eqs. (9) and (10), the limit exists in the norm, and

$$
\|A\|=\sup _{X}|A(X)| .
$$

The classical algebra of observables can be considered as the $C^{*}$-algebra $\mathscr{C}(K)$ of continuous functions on $K$, the family of all subsets of $Z$, with respect to the following topology. To specify a set $X \subset Z$, one has to determine for any point $x \in Z$ if it belongs to $X$ or not. In that sense

$$
K=\bigotimes_{x \in Z}\{0,1\}_{x}
$$


Now take on each set $\{0,1\}_{x}$ the discrete topology, and on $K$ the associated direct product topology. According to Tychonov's theorem, $K$ is compact.

In this topology, two sets $X$ and $Y$ are "close to" each other, if they have the same intersection with some "large", but finite set $\Lambda \subset Z$. Therefore, Eq. (10) expresses the continuity of $A(X)$.

\section{States}

As usual, a state will be a positive linear form of norm 1 on the algebra of observables. A state $\omega$ on the quantum algebra $\mathfrak{a}$ is called a classical state, if it is unequal to zero only on the classical algebra. Equivalently,

$$
\omega\left(a^{*}(X) a(Y)\right)=0 \quad \text { if } \quad X \neq Y .
$$

A classical state $\omega$ on $\mathfrak{a}$ defines a state on the classical algebra $\mathscr{C}(K)$, that is a measure on $K$. We have [7]

$$
\omega(A)=\int \mu(d X) A(X)=\lim _{X \rightarrow \infty} \sum_{X \subset A} \mu_{\Lambda}(X) A(X) .
$$

The second equality can be regarded as the definition of the integral as a limit of Riemannian sums. Corresponding to a finite $\Lambda$, the set $K$ is divided into $2^{N(\Lambda)}$ intervals of the form

$$
I_{\Lambda}(X)=\{Y \in K: Y \cap \Lambda=X\} .
$$

The measure attached to the interval $I_{\Lambda}(X)$ is

$$
\mu_{\Lambda}(X)=\int_{I_{\Lambda}(X)} \mu\left(d Y^{\prime}\right)=\int_{I_{\Lambda}(X)} \mu_{\Lambda}(X, d Y) ;
$$

it is often convenient to write

with

$$
\mu\left(d Y^{\prime}\right)=\mu_{A}(X, d Y),
$$

$$
Y^{\prime}=X \cup Y, X \subset \Lambda, Y \subset Z / \Lambda .
$$

Eq. (14) yields the interpretation of $\mu_{\Lambda}(X)$; it is the probability that the points in $X$ are occupied, whereas the other points of $\Lambda$ are empty. In terms of the original state $\omega$,

$$
\mu_{\Lambda}(X)=\omega\left(a^{*}(X) a(X) a(\Lambda / X) a^{*}(\Lambda / X)\right) .
$$

By Eq. (12), the state $\omega$ is uniquely determined by all quantities $\mu_{\Lambda}(X)$. It follows from Eq. (14) that they must satisfy the consistency 
relations

$$
\begin{gathered}
\sum_{X \subset A} \mu_{\Lambda}(X)=1, \\
\sum_{Y \subset M_{\mid \Lambda}} \mu_{M}(X \cup Y)=\mu_{\Lambda}(X) .
\end{gathered}
$$

Therefore $\omega$ is already determined by its correlation functions

$$
\varrho(X)=\mu_{X}(X)=\omega\left(a^{*}(X) a(X)\right) .
$$

With $\varrho(\emptyset)=1$, we have [6]

$$
\mu_{\Lambda}(X)=\sum_{X \subset Y \subset A} \varrho(Y)(-1)^{N(Y)-N(X)} .
$$

Remark that this equality corresponds to

$$
a^{*}(X) a(X) a(\Lambda / X) a^{*}(\Lambda / X)=\sum_{X \subset Y \subset \Lambda} a^{*}(Y) a(Y)(-1)^{N(Y)-N(X)} .
$$

\section{Equilibrium States}

Following Lanford and Ruelle [7] and Dobrushin [12, 13], we will say that a classical state satisfies the equilibrium condition (E 1) with respect to the interaction $\Phi$, if the corresponding measure satisfies

$$
\begin{aligned}
& \mu_{\Lambda}(X \cup\{x\}, d Y)=\mu_{\Lambda}(X, d Y) \exp [-U(X \cup\{x\} \cup Y)+U(X \cup Y)], \\
& \text { for all finite } \Lambda, x \in \Lambda, X \subset \Lambda /\{x\}, Y \subset Z / \Lambda \text {. }
\end{aligned}
$$

This condition is plausible from the Gibbs grand canonical prescription; as mentioned before, the inverse temperature factor $\beta$ and the chemical potential term $-\mu$ have been absorbed in the interaction.

Lemma 1. The function

$$
\begin{aligned}
B_{x}(X)=\sum_{S \subset X \cup\{x\}} \Phi(S) & =U(X)-U(X /\{x\}), & & x \in X \\
& =U(X \cup\{x\})-U(X), & & x \notin X
\end{aligned}
$$

is continuous. So is its exponential.

Proof. We have

$$
\left|B_{x}(X)-B_{x}(X \cap \Lambda)\right|=\left|\sum_{\substack { S \subset X \cup\{x\} \\
\begin{subarray}{c}{x \in S \\
S \notin \Lambda{ S \subset X \cup \{ x \} \\
\begin{subarray} { c } { x \in S \\
S \notin \Lambda } }\end{subarray}} \Phi(S)\right| \leqq \sum_{\substack{x \in S \\
S \notin \Lambda}}|\Phi(S)|,
$$

so $B_{x}(X)$ is continuous (Eqs. (1) and (10)). 
Let us remark here that the terms $U$ in Eqs. (20) and (21) are not necessarily finite separately. However, their difference, as defined by Eq. (21) is finite by Eq. (1).

For the case of a translationally invariant interaction $\Phi$, Lanford and Ruelle [7] proved the following theorem.

Theorem 2. Let $\Phi$ be invariant. Then an invariant state satisfies (E1) precisely if it is an invariant equilibrium state.

The notion of "invariant equilibrium state" is introduced in [7]; it is an invariant state that maximizes the difference of mean entropy and mean energy, thus giving the pressure [5].

Let us give now a condition equivalent to (E 1).

Theorem 3. A state satisfies (E1) precisely if

$$
\begin{aligned}
& \mu_{\Lambda}(X \cup\{x\})=\int_{I_{\Lambda}(X)} \mu_{\Lambda}(X, d Y) \exp [-U(X \cup\{x\} \cup Y)+U(X \cup Y)], \\
& \text { (E2) for all finite } \Lambda, x \in \Lambda, X \subset \Lambda /\{x\} .
\end{aligned}
$$

Proof. It is clear that (E2) follows from (E1) by integration over all $Y \subset Z / \Lambda$. This is allowed because of the continuity of the exponential, lemma 1 . Now assume that (E2) is satisfied. Take any $M \supset \Lambda, Y_{1} \subset M / \Lambda$, and consider the interval

Then

$$
I_{M}\left(X \cup Y_{1}\right) \subset I_{A}(X) \subset K .
$$

$$
\begin{aligned}
& \int_{I_{M}\left(X \cup\{x\} \cup Y_{1}\right)} \mu_{A}(X \cup\{x\}, d Y)=\mu_{M}\left(X \cup\{x\} \cup Y_{1}\right) \\
\quad= & \int_{I_{M}\left(X \cup Y_{1}\right)} \mu_{M}\left(X \cup Y_{1}, d Y_{2}\right) \exp \left[-U\left(X \cup\{x\} \cup Y_{1} \cup Y_{2}\right)+U\left(X \cup Y_{1} \cup Y_{2}\right)\right] \\
= & \int_{I_{M}\left(X \cup Y_{1}\right)} \mu_{\Lambda}(X, d Y) \exp [-U(X \cup\{x\} \cup Y)+U(X \cup Y)],
\end{aligned}
$$

where Eq. (14) and (E 2) have been used. The equality of the first and last members for any interval $I_{M}\left(X \cup Y_{1}\right) \subset I_{\Lambda}(X)$ implies, with Eq. (12), that

$$
\begin{aligned}
& \int_{I_{\Lambda}(X \cup\{x\})} \mu_{\Lambda}(X \cup\{x\}, d Y) A(Y) \\
& =\int_{I_{\Lambda}(X)} \mu_{\Lambda}(X, d Y) A(Y) \exp [-U(X \cup\{x\} \cup Y)+U(X \cup Y)]
\end{aligned}
$$

for any continuous function $A(Y)$, which is equivalent to (E1). This proves Theorem 3.

If the interaction $\Phi$ is of finite range, Eq. (4), the condition (E2) reduces to a very simple form. 
Theorem 4. A state satisfies (E2) with respect to a finite range interaction $\Phi$ precisely if

$$
\begin{aligned}
& \mu_{\Lambda}(X \cup\{x\})=\mu_{\Lambda}(X) \exp [-U(X \cup\{x\})+U(X)] \\
& \text { for all } x \in Z, \Lambda \supset \Lambda(x), X \subset \Lambda /\{x\} .
\end{aligned}
$$

Proof. If $\Lambda \supset \Lambda(x), X \subset \Lambda /\{x\}, Y \subset Z / \Lambda$,

$$
U(X \cup\{x\} \cup Y)-U(X \cup Y)=U(X \cup\{x\})-U(X) .
$$

Then (EFR) immediately follows from (E2).

Now take any finite $\Lambda$, and $x \in \Lambda, X \subset \Lambda /\{x\}, Y \subset Z / \Lambda$. Let $M=\Lambda \cup \Lambda(x)$. Then, with Eq. (16) and (EFR),

$$
\begin{aligned}
\mu_{\Lambda}(X \cup\{x\}) & =\sum_{Y_{1} \subset M / \Lambda} \mu_{M}\left(X \cup\{x\} \cup Y_{1}\right) \\
& =\sum_{Y_{1} \subset M / \Lambda} \mu_{M}\left(X \cup Y_{1}\right) \exp \left[-U\left(X \cup\{x\} \cup Y_{1}\right)+U\left(X \cup Y_{1}\right)\right] \\
& =\int_{I_{\Lambda}(X)} \mu_{\Lambda}(X, d Y) \exp [-U(X \cup\{x\} \cup Y)+U(X \cup Y)] .
\end{aligned}
$$

By Eq. (24), the integrand depends only on $Y_{1}=Y \cap M$, which justifies the last equality. This proves Theorem 4.

Restricting condition (E2), Eq. (22) to $\Lambda=X \cup\{x\}$, we obtain the following.

$$
\varrho(X \cup\{x\})=\int_{I_{X} \cup\{x\}(X)} \mu_{X \cup\{x\}}(X, d Y) \exp [-U(X \cup\{x\} \cup Y)+U(X \cup Y)]
$$

for all finite $X, x \notin X$.

It will be shown in Section 6 that both (E2) and (E3) are equivalent to the KMS boundary condition.

\section{Time Translations}

In order to come to time translations, we assign to each finite set $\Lambda$ a Hamiltonian $H(\Lambda) \in \mathfrak{a}(\Lambda)$ (cf. [3]). It must satisfy

$$
\left(a^{*}(X) \Omega, H(\Lambda) a^{*}(Y) \Omega\right)=U(X) \delta_{X Y},
$$

for any $X, Y \subset A$. That is

$$
H(\Lambda)=\sum_{X \subset A} U(X) a^{*}(X) a(X) a(\Lambda / X) a^{*}(\Lambda / X) .
$$

Because $H(\Lambda)$ is a diagonal matrix in the representation (6), it is immediately clear that

$$
\exp [i t H(\Lambda)]=\sum_{X \subset A} \exp [i t U(X)] a^{*}(X) a(X) a(\Lambda / X) a^{*}(\Lambda / X) .
$$


Let us show now that the above sequence of Hamiltonians defines a group of automorphisms on the quantum algebra of observables $\mathfrak{a}$. The limit

$$
\lim _{\Lambda \rightarrow \infty} A_{t}^{(\Lambda)}=\lim _{\Lambda \rightarrow \infty} \exp [i t H(\Lambda)] A \exp [-i t H(\Lambda)] .
$$

will be proved to exist for any $A \in \mathfrak{a}$.

First consider $A=a^{*}(x)$, the creation operator in the point $x$.

Lemma 5. For any $\Lambda$ containing $x$,

$$
\begin{aligned}
& a^{*}(x)_{t}^{(\Lambda)} \\
& =a^{*}(x) \sum_{Y \subset \Lambda /\{x\}} \exp \{i t[U(Y \cup\{x\})-U(Y)]\} a^{*}(Y) a(Y) a(\Lambda /\{x\} / Y) a^{*}(\Lambda /\{x\} / Y) .
\end{aligned}
$$

The limit

$$
a^{*}(x)_{t}=\lim _{\Lambda \rightarrow \infty} a^{*}(x)_{t}^{(\Lambda)}
$$

exists in the norm for any complex $t$. The operator $a^{*}(x)_{t}$ is norm analytic in the complex t-plane.

Proof. Using the expression (27) for $\exp [i t H(\Lambda)]$, one immediately obtains Eq. (29). We can write

$$
a^{*}(x)_{t}^{(\Lambda)}=a^{*}(x) \exp \left[i t B_{x}^{(\Lambda)}\right],
$$

where $B_{x}^{(\Lambda)}$ is the classical observable corresponding to the function $B_{x}(X \cap \Lambda)$, Eq. (21). The continuity of $B_{x}(X)$, Lemma 1, implies the existence of the limit (30). Clearly the operators $a^{*}(x)_{t}^{(1)}$ are norm analytic. Because the limit (30) is uniform in any bounded region of the complex $t$-plane, it follows that $a^{*}(x)_{t}$ is analytic. This proves Lemma 5 .

The time translation for observables in one point $x$ is now given by

$$
\begin{gathered}
a^{*}(x)_{t}=a^{*}(x) \exp \left[\text { it } B_{x}\right], a(x)_{t}=a(x) \exp \left[-i t B_{x}\right] \\
{\left[a^{*}(x) a(x)\right]_{t}=a^{*}(x) a(x), \quad\left[a(x) a^{*}(x)\right]_{t}=a(x) a^{*}(x) .}
\end{gathered}
$$

It is clear then, that the limit (28) exists for any local observable and any complex $t$.

Let us define $\hat{\mathfrak{a}}(\Lambda)$ as the algebra spanned by observables of the form $A B$, where $A \in \mathfrak{a}(\Lambda)$ and $B$ is any classical observable. Let further

$$
\hat{\mathfrak{a}}_{L}=\bigcup_{\Lambda} \hat{\mathfrak{a}}(\Lambda) .
$$

It follows from Eq. (31), that the algebras $\hat{\mathfrak{a}}(\Lambda)$ are mapped onto themselves for any complex $t$. We have the following theorem. 
Theorem 6. For any $A \in \hat{\mathfrak{a}}_{L}$ and any complex $t$, the limit (28) exists and defines a norm analytic operator $A_{t}$. The mapping $A \rightarrow A_{t}$ defines a group of automorphisms on any $\hat{\mathfrak{a}}(\Lambda)$ and on $\hat{\mathfrak{a}}_{L}$.

For any $A \in \mathfrak{a}$ and any real $t$, the limit (28) exists. The mapping $A \rightarrow A_{t}$ defines a strongly continuous group of *automorphisms on $\mathfrak{a}$.

Note that the time translation of $A \in \mathfrak{a}$ in general can only be defined for real $t$. If $A_{n} \rightarrow A$, the limit

$$
\lim _{n \rightarrow \infty} A_{n t}^{(\Lambda)}=A_{t}^{(\Lambda)}
$$

is uniform in $\Lambda$ only for real $t$.

Let us conclude this section with the following remark. Instead of the Hamiltonian $H(\Lambda)$, Eq. (26), one can take for any configuration $S \subset Z$ the Hamiltonian

$$
H(\Lambda, S)=\sum_{X \subset \Lambda}\left[U\left(X \cup S_{1}\right)-U\left(S_{1}\right)\right] a^{*}(X) a(X) a(\Lambda / X) a^{*}(\Lambda / X) .
$$

Here, $S_{1}=S \cap(Z / \Lambda)$. This Hamiltonian leads to

$$
\begin{aligned}
a^{*}(x)_{t}^{(\Lambda, S)}= & a^{*}(x) \sum_{Y \subset \Lambda /\{x\}} \exp \left\{i t\left[U\left(Y \cup\{x\} \cup S_{1}\right)-U\left(Y \cup S_{1}\right)\right]\right\} \\
& \times a^{*}(Y) a(Y) a(\Lambda /\{x\} / Y) a^{*}(\Lambda /\{x\} / Y)
\end{aligned}
$$

However, the time translation automorphism, Theorem 6, is independent of $S$.

$$
\lim _{\Lambda \rightarrow \infty} A_{t}^{(\Lambda, S)}=\lim _{\Lambda \rightarrow \infty} A_{t}^{(\Lambda)}=A_{t}
$$

for $A \in \hat{\mathfrak{a}}_{L}, t$ complex, and for $A \in \mathfrak{a}, t$ real.

\section{The KMS Boundary Condition}

A state $\omega$ on the algebra $a$ will be said to satisfy the KMS boundary condition with respect to the interaction $\Phi$ if (cf. $[8,3]$ )

i.

$$
\begin{aligned}
& \int d t f(t) \omega\left(B_{t} A\right)=\int d t f(t-i) \omega\left(A B_{t}\right) \\
& \text { for all } A, B \in \mathfrak{a}, \hat{f} \in \mathscr{D} .
\end{aligned}
$$

For classical interactions, so that the time translation has the analyticity properties of Theorem 6 , one can write down without trouble the equivalent conditions

ii.

$$
\omega\left(B_{t} A\right)=\omega\left(A B_{t+i}\right) \quad \text { for all } A \in \mathfrak{a}, B \in \mathfrak{a}_{L},
$$

iii.

$$
\omega\left(a^{*}(x) A\right)=\omega\left(A a^{*}(x)_{i}\right) \text { for all } A \in \mathfrak{a}_{L}, x \in Z \text {. }
$$

For the equivalence of i. and ii., see [8]. Let us indicate how iii. implies ii. 
Going over to the adjoint operators in iii., one gets

Then

$$
\omega(a(x) A)=\omega\left(A a(x)_{i}\right) \text { for all } \quad x \in Z, A \in \mathfrak{a}_{L} .
$$

$$
\omega\left(a^{*}(x) a(x) A\right)=\omega\left(A a^{*}(x) a(x)\right) .
$$

From this it follows that $\omega$ is a classical state. For example, let $x \in X, x \notin Y$. Then

$$
\begin{aligned}
\omega\left(a^{*}(X) a(Y)\right) & =\omega\left(a^{*}(x) a(x) a^{*}(x) a^{*}(X /\{x\}) a(Y)\right) \\
& =\omega\left(a^{*}(x) a^{*}(X /\{x\}) a(Y) a^{*}(x) a(x)\right)=0 .
\end{aligned}
$$

We have that

$$
\omega(B A)=\omega\left(A B_{i}\right),
$$

if $A \in \mathfrak{a}_{L}$ and $B$ is located in one point. Then (35) follows for any $B \in \mathfrak{a}_{L}$, and because $\omega$ is classical, for any $B \in \hat{\mathfrak{a}}_{L}$. This yields condition ii.

We now come to the main theorem.

Theorem 7. For a state $\omega$ on a the following conditions, all with respect to the classical interaction $\Phi$, are equivalent.

1. $\omega$ satisfies $K M S$,

2. $\omega$ is classical and satisfies (E 2), Eq. (22),

3. $\omega$ is classical and satisfies (E3), Eq. (25).

Proof. The implication $2 \Rightarrow 3$ is trivial. Let us prove $1 \Rightarrow 2$. We already saw, that a KMS state must be classical. Applying the KMS condition to $\mu_{A}(X \cup\{x\})$, we find

$$
\begin{aligned}
\mu_{\Lambda}(X \cup\{x\}) & =\omega\left(a^{*}(x) a(x) a^{*}(X) a(X) a(\Lambda /\{x\} / X) a^{*}(\Lambda /\{x\} / X)\right) \\
& =\omega\left(a(x) a^{*}(x)_{i} a^{*}(X) a(X) a(\Lambda /\{x\} / X) a^{*}(\Lambda /\{x\} / X)\right) .
\end{aligned}
$$

Substituting Eq. (29), we obtain

$$
\mu_{\Lambda}(X \cup\{x\})=\lim _{M \rightarrow \infty} \sum_{Y \subset M / A} \mu_{M}(X \cup Y) \exp [-U(X \cup\{x\} \cup Y)+U(X \cup Y)],
$$

which is (E 2).

Let us finally show that 3 implies 1 . Because $\omega$ is classical, we have that

$$
\omega\left(a^{*}(x) a^{*}(X) a(Y)\right)=\omega\left(a^{*}(X) a(Y) a^{*}(x)_{i}\right)=0,
$$

unless $x \notin X, Y=X \cup\{x\}$. But in that case we have from (E 3) and the argument above reversed, that

$$
\omega\left(a^{*}(x) a(x) a^{*}(X) a(X)\right)=\omega\left(a(x) a^{*}(X) a(X) a^{*}(x)_{i}\right) .
$$


With Eq. (19), this proves that

$$
\omega\left(a^{*}(x) A\right)=\omega\left(A a^{*}(x)_{i}\right),
$$

for any local observable $A$. This concludes the proof.

\section{The Limit of Finite Volume States}

We will show in this section that a limit of finite volume Gibbs states satisfies the KMS condition.

Let a configuration $S \subset Z$ and a finite volume $M$ be given. Then the state $\omega^{(M, S)}$ is defined as follows

For $\Lambda \subset M, X \subset \Lambda$,

$$
\begin{aligned}
\mu_{\Lambda}^{(M, S)}(X)= & \left\{\sum_{Y \subset M} \exp \left[-U\left(Y \cup S_{1}\right)+U\left(S_{1}\right)\right]\right\}^{-1} \\
& \times \sum_{Y \subset M / \Lambda} \exp \left[-U\left(X \cup Y \cup S_{1}\right)+U\left(S_{1}\right)\right] .
\end{aligned}
$$

Here $S_{1}=S \cap(Z / M)$. For $\Lambda^{\prime} \subset Z / M, X^{\prime} \subset \Lambda^{\prime}$, we define

$$
\begin{array}{rll}
\mu_{\Lambda \cup \Lambda^{\prime}}^{(M, S)}\left(X \cup X^{\prime}\right)=\mu_{\Lambda}^{(M, S)}(X), & \text { if } & X^{\prime}=S \cap \Lambda^{\prime} ; \\
0, & \text { if } & X^{\prime} \neq S \cap \Lambda^{\prime} .
\end{array}
$$

With this definition and Eq. (33), we have

$$
\omega^{(M, S)}(A)=\{\operatorname{Tr}[\exp (-H(M, S))]\}^{-1} \operatorname{Tr}[A \exp (-H(M, S))],
$$

for any $A \in \mathfrak{a}(M)$.

Let us consider the set of states $\omega^{(M, S)}$ for $S$ fixed and for any $M$. It is a subset of the unit ball of the dual space of $\mathscr{C}(K)$ (Section 2). Because $K$ is compact, the unit ball in $\mathscr{C}(K)^{*}$ is $w^{*}$-compact $([15]$, Ch. $3, \S 12.7)$. Therefore there exists at least one state $\omega$, which is the $w^{*}$-limit of some sequence $\omega^{\left(M_{n}, S\right)}$. That is,

$$
\mu_{\Lambda}(X)=\lim _{n \rightarrow \infty} \mu_{\Lambda}^{\left(M_{n}, S\right)}(X) \text { for any } \Lambda, X \in \Lambda .
$$

The limit may depend on the subsequence $M_{n}$ as well as on the configuration $S([16,11,20,18] ;[13,14])$.

Theorem 8. Any limit, in the sense of Eq. (39), of Gibbs states, Eqs. $(36,37)$, satisfies the KMS condition.

Proof. With Eqs. (34) and (38), one has

$$
\omega^{(M, S)}\left(a^{*}(x) A\right)=\omega^{(M, S)}\left(A a^{*}(x)_{i}^{(M, S)}\right),
$$


for any $A \in \mathfrak{a}(M)$. Taking the subsequence $M_{n} \rightarrow \infty$, one obtains the KMS condition (cf. [8], p. 225-227). This concludes the proof.

The above considerations immediately lead to the following result.

Theorem 9. The KMS condition and the equivalent conditions (E1-3) are satisfied by at least one state.

It is an interesting question, if the converse of Theorem 8 is true. That is, is any KMS-state a limit of Gibbs states, in the sense of Eq. (39)?

\section{Equations for the Correlation Functions}

With the help of Eqs. (12) and (18), rewrite the right member of (E 3), Eq. (25). The result is

$$
\begin{aligned}
& \varrho(X \cup\{x\})=\sum_{T \subset Z)}[\varrho(X \cup T)-(X)-\varrho(X \cup\{x\} \cup T)] K(x \cup\{x\}, X, T) \\
& \text { for all finite } \quad X, x \notin X .
\end{aligned}
$$

The quantity $K$ is defined as

$$
K\left(X, X_{1}, T\right)=\sum_{Y \subset T}(-1)^{N(T)-N(Y)} \exp \left[-U(X \cup Y)+U\left(X_{1} \cup Y\right)\right] .
$$

We can enlarge the set of equations (E4) as follows.

$$
\begin{aligned}
& \varrho(X)=\sum_{T \subset Z / X}\left[\sum_{S \subset X / X_{1}}(-1)^{N(S)} \varrho\left(X_{1} \cup S \cup T\right)\right] K\left(X, X_{1}, T\right), \\
& \text { for all finite } X \neq \emptyset, X_{1} \subset X, X_{1} \neq X .
\end{aligned}
$$

If condition (E 1), Eq. (20) is written in the form

$$
\begin{aligned}
& \mu_{\Lambda}(X, d Y)=\mu_{\Lambda}\left(X_{1}, d Y\right) \exp \left[-U(X \cup Y)+U\left(X_{1} \cup Y\right)\right] \\
& \text { for all finite } \Lambda, X \subset \Lambda, X_{1} \subset \Lambda, Y \subset Z / \Lambda,
\end{aligned}
$$

the full set of equations (E 5) can be derived from it in the same way as (E4) has been derived from (E1). The equivalence of (E4) and (E1) then yields the equivalence of (E 4) and (E 5).

An open question is, how many of the equations of the set (E5) can be omitted at most. In other words, what are the minimal subsets of (E 5) that are equivalent to the full set (E 5).

One might expect that such a minimal subset is obtained if one takes for any finite $X \neq \emptyset$ some fixed $X_{1} \subset X, X_{1} \neq X$, instead of all such $X_{1}$. Then, loosely speaking, the number of equations and the number of unknown quantities $\varrho(X)$ are equal. (If $Z$ is finite, this is true.)

If one chooses $X_{1}$ such, that it contains one point less than $X$, one obtains the equations of Gallavotti and Miracle-Sole ([9]; [1], p. 82). 
For two-body interactions, they reduce to the lattice analogue of the Kirkwood-Salsburg equations ([7]; [1], p. 72).

For two-body interactions $\Phi(x, y)$, Eq. (41) becomes

$$
K\left(X, X_{1}, T\right)=\exp \left[-U(X)+U\left(X_{1}\right)\right] \prod_{y \in T}\left\{-1+\prod_{x \in X / X_{1}} \exp [-\Phi(x, y)]\right\} .
$$

If one defines $\Phi(x, x)=+\infty$, Eq. (42) becomes

$$
\varrho(X)=\exp \left[-U(X)+U\left(X_{1}\right)\right] \sum_{Y \subset \boldsymbol{Z} / X_{1}} \varrho\left(X_{1} \cup Y\right) \prod_{y \in Y}\left\{-1+\prod_{x \in X / X_{1}} \exp [-\Phi(x, y)]\right\} .
$$

A particular choice of $X_{1}, N(X)-N\left(X_{1}\right)=1$, gives the Kirkwood-Salsburg equations. The choice $X_{1}=\emptyset$ leads to the Mayer-Montroll equations ([1], p. 79).

In general, the important merit of the Kirkwood-Salsburg and Gallavotti-Miracle equations is, that they admit a unique solution if the temperature is high enough. Such a result of Gallavotti and MiracleSole [9] applies to translationally invariant potentials. Here, it remains valid for interactions which are bounded in the sense of Eq. (2).

However, if the Gallavotti-Miracle equations have a unique solution, the conditions (E1-5) and the KMS-condition must have the same unique solution (Section 7). Furthermore, this unique state is the limit of Gibbs states, Eq. (39), independent of the subsequence $M_{n}$ and the configuration $S$.

Let us finally mention a result for the quantum lattice gas. A generalization of the Gallavotti-Miracle equations has been found by Greenberg [22] and Robinson. It has been shown by Lanford [23] that these equations can also be derived from the KMS condition.

\section{Conclusion}

The remarkable fact that we can do without lattice translations, is due to the fact that only the state is considered. As soon as one desires thermodynamical quantities as the mean entropy and the global pressure, there has to be some sort of translational invariance. For a lattice without translations, see also the papers by Griffiths [17] and Kelly and Sherman [19] on ferromagnetic interactions.

In the main part of the present paper (Sections 4-6) the equivalence of different equilibrium conditions (KMS etc.) has been proved. In Sections 7 and 8, the connection with Gibbs states and with the Gallavotti-Miracle equations has been given. We have

$$
\text { Limit of Gibbs states } \Rightarrow \text { KMS etc. } \Rightarrow \text { Gallavotti-Miracle eqs. }
$$


If the Gallavotti-Miracle equations have a unique solution (high temperatures), the implications in the opposite direction are also true. It is an open question, if these implications can generally be proved.

In a separate paper [21], we will treat the one dimensional lattice gas with finite range interactions. It will be shown, that the KMScondition and the variational principle lead to a unique equilibrium state, which can be calculated explicitly.

Acknowledgments. The work described in this paper is part of the research program of the "Foundation for Fundamental Research on Matter" (F.O.M.), which is financially supported by the "Netherlands Organization for Pure Research" (Z.W.O.). The author wishes to thank Professor N. M. Hugenholtz for his stimulating help.

\title{
References
}

1. Ruelle, D.: Statistical mechanics. New York-Amsterdam: Benjamin 1969.

2. Robinson, D. W.: Commun. Math. Phys. 6, 151 (1967).

3. - Commun. Math. Phys. 7, 337 (1968).

4. Gallavotti, G., Miracle-Sole, S.: Commun. Math. Phys. 5, 317 (1967).

5. Ruelle, D.: Commun. Math. Phys. 5, 324 (1967).

6. - In: Fundamental problems in statistical mechanics, ed. E. G. D. Cohen. Amsterdam: North Holland 1968.

7. Lanford, O. E., Ruelle, D.: Commun. math. Phys. 13, 194 (1969).

8. Haag, R., Hugenholtz, N. M., Winnink, M.: Commun. Math. Phys. 5, 215 (1967).

9. Gallavotti, G., Miracle-Sole, S.: Commun. Math. Phys. 7, 274 (1968).

10. Manuceau, J., Trotin, J. C.: Ann. Inst. Henri Poincaré, A 10, 359 (1969).

11. Dobrushin, R. L.: Theory of probability and its applications 10, 193 (1965).

12. - Theory of probability and its applications 13, 197 (1968).

13. - Funkts. Analiz i ego Pril. 2, 31, 44 (1968).

14. Ginibre, J.: On some recent work of Dobrushin, Colloque International du C.N.R.S., Mai 1969, Gif-sur-Yvette.

15. Choquet, G.: Lectures on analysis, vol. I. New York-Amsterdam: Benjamin 1969.

16. Griffiths, R. B.: Phys. Rev. 136, A 437 (1964).

17. - J. Math. Phys. 8, 478, 484 (1967).

18. -- Commun. Math. Phys. 6, 121 (1967).

19. Kelly, D. G., Sherman, S.: J. Math. Phys. 9, 466 (1968).

20. Ginibre, J., Grossman, A., Ruelle, D.: Commun. Math. Phys. 3, 187 (1966).

21. Brascamp, H. J.: In preparation.

22. Greenberg, W.: Commun. Math. Phys. 11, 314 (1969).

23. Lanford, O. E.: High temperature expansions, Cargèse Summerschool 1969.

\author{
H. J. Brascamp \\ Institute for Theoretical Physics \\ Hoogbouw WSN \\ Universiteitscomplex Paddepoel \\ Groningen, Netherlands
}

